\title{
IP Multimedia Subsystem: Overview and Services
}

\author{
Jegede Olawale \\ LM Ericsson Nigeria Ltd., \\ Nigeria
}

jegede1@yahoo.com

Shokunbi Opeyemi Nestle Nigeria PLC, Nigeria

opeshokunbi@yahoo.ca

\author{
Onyenweaku Edward \\ New Horizons Nigeria Ltd., \\ Nigeria
}

edynol@yahoo.com

Awodele Oludele

Babcock University, Nigeria

delealways@yahoo.com

\begin{abstract}
This work presents IP Multimedia Subsystem and its emerging applications in the field of Telecommunication and Information Technology. We have examined the standard architecture of IMS based on the Third Generation Partnership Project (3GPP) and the basic operation which is being modified for different solutions by different vendors.

The goal is to examine the applications of IMS along with the capabilities and/or benefits IMS brings to the mobile network operators and end users in terms of new services and the overall experience. We concluded by identifying present limitations of IMS while also discussing the future.
\end{abstract}

Keywords: IP Multimedia Subsystem (IMS), Quality of Service (QoS).

\section{Introduction}

Multimedia services have emerged over the years and proved to be the future of telecommunication, a result of which compelled operators and vendors to create a total solution to harness the multimedia opportunities. This total solution is the IP Multimedia Subsystem (IMS). IMS is a label that has started springing up all over the telecom industry, whether on individual products, or as vendor systems platforms or interoperability programs. Though initially very complicated and pretty abstract, it has become a reality and is perceived to be the big vision of the future of telecommunication (Telecom Report, 2005).

Material published as part of this publication, either on-line or in print, is copyrighted by the Informing Science Institute. Permission to make digital or paper copy of part or all of these works for personal or classroom use is granted without fee provided that the copies are not made or distributed for profit or commercial advantage AND that copies 1) bear this notice in full and 2) give the full citation on the first page. It is permissible to abstract these works so long as credit is given. To copy in all other cases or to republish or to post on a server or to redistribute to lists requires specific permission and payment of a fee. Contact 0HPublisher@InformingScience.org to request redistribution permission.
IP Multimedia Subsystem (IMS) is defined by $3 \mathrm{GPP}$ as a new subsystem, i.e. a new mobile network infrastructure that enables the convergence of data, speech and mobile network technology over an IP-based infrastructure. More importantly it represents a $3 \mathrm{GPP}$ and $3 \mathrm{GPP} 2$ effort to define and create an all IP based wireless network as compared to the historically disparate voice, data, 
signaling, and control network elements (Mangini, 2006).

IMS is a solution designed to fill the gap between the existing traditional telecommunications technology and Internet Technology (I.T.) that increased bandwidth alone will not provide. It was specifically architected to enable and enhance real time, multimedia mobile services such as rich voice services, video telephony, messaging, conferencing, and push services. IMS enables these user-to-user communication services via a number of key mechanisms including Session Negotiation and Management, QoS and Mobility Management. (3G Americas).

\section{The Search for Success-Service Sales}

Technology convergence, regulatory changes, competitive pressures and maturing markets are continuously changing the future for wireless telecommunication providers. The most promising source of relief is found in multimedia services and IMS has been able to address the following issue:

- A common platform with reusable components, providing quick and easy service development.

- Decreased deployment and management costs: Mobile network operators can experience decreased CAPEX through sharing components and integration costs, as well as decreased OPEX through lower complexity for the MNO.

- Consistent, open interfaces for $3^{\text {rd }}$ party developers. IMS entities (CSCF, HSS, MRF, etc.) are standardized in terms of functionality as well as external interfaces.

- Consistency to end users: Roaming scenarios are possible, not restricting access to a service to the home network only (Motorola, 2005).

\section{IP Platform}

IP-based systems offer network operators the opportunity to expand their services, integrating voice and multimedia communications and delivering them into new environments with new purposes. This is what the industry calls convergence, bringing multiple media, multiple points of access, and multiple modes of and purposes for communication together into a single network, and often, even into a single device. Developing and delivering convergence, though, will make a number of new demands on both the network and the operator.

IP-based packet switched technology is the backbone of the desired Multimedia experience and is being supported as the most direct path to operator success in the future. Market analysts predicted that by 2005, users of packet switched services will account for nearly half of the worldwide installed user base... and by 2008, will outnumber circuit switched (voice only) users by nearly four to one; this has been the case to a large extent.

The IMS Report of October 2003 projects that within several years, packet switched data services will represent the majority of the wireless marketplace.

\section{IMS Architecture}

IMS started as a technology for 3G mobile networks (under the auspices of the 3rd Generation Partnership Project (3GPP)), but it is now spreading to next-generation wireline networks and has been a key to fixed/mobile convergence. It builds on the Session Initiation Protocol (SIP), which has emerged as the crucial technology for controlling communications in IP-based NextGeneration Networks (NGNs). 


\section{IMS Solutions}

Leading Telecommunication and Information Technology Vendors (such as Motorola, Ericsson, Cisco, Metaswitch, IBM, Nortel, etc) have developed different IMS solutions for the MNOs. The various solutions turn the Intelligent Network into an Intuitive Network that is device, application and access aware. With IMS, vendors deliver empowered multimedia services that provide new levels of personalization, security and mobility (Nortel, 2006).

A quick view of a typical architecture is shown in Figure 1 and explained courtesy of Metaswitch Networks.

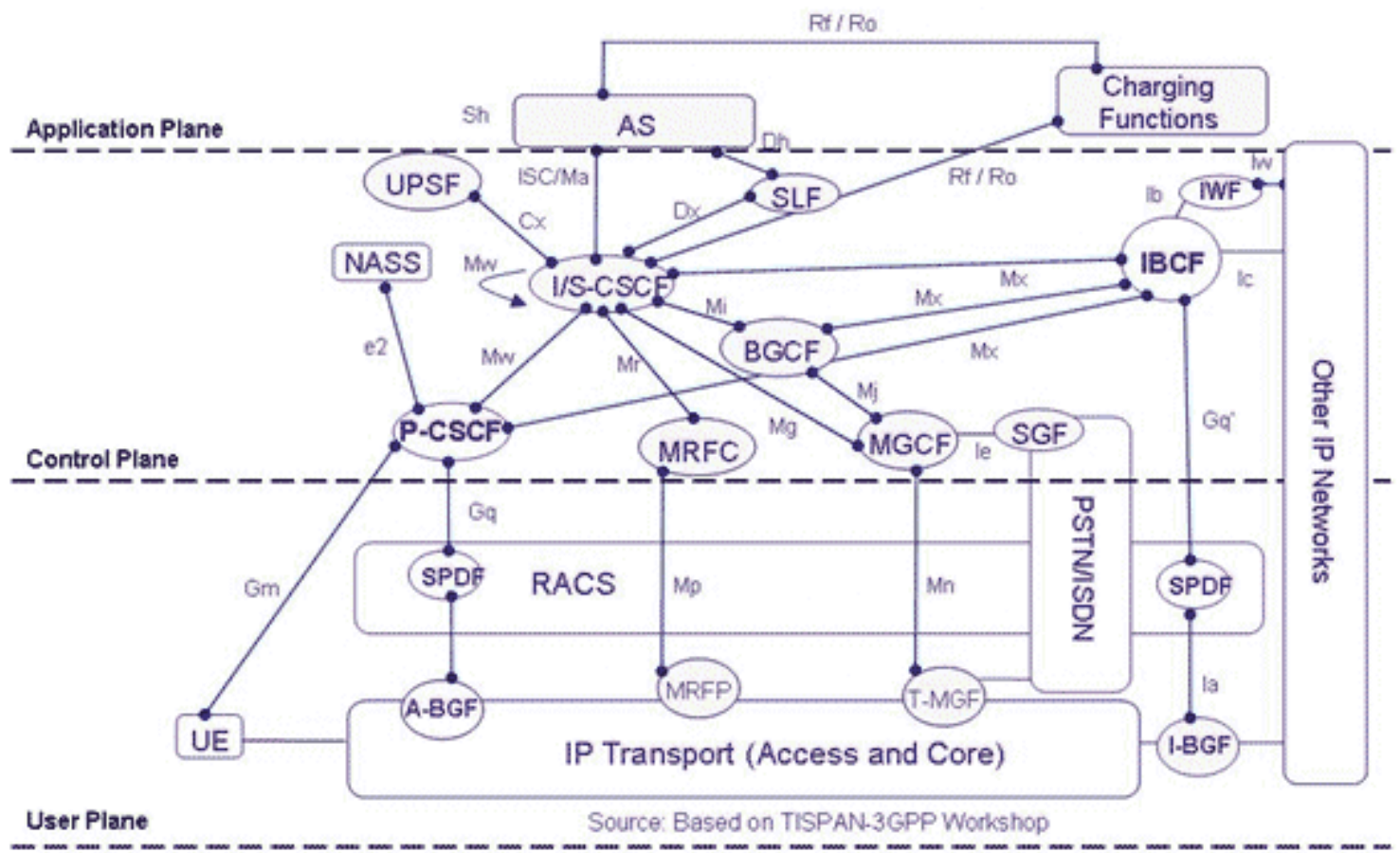

Figure 1. Typical Architecture (from Metaswitch Networks)

IMS decomposes the networking infrastructure into separate functions with standardized interfaces between them. Each interface is specified as a "reference point", which defines both the protocol over the interface and the functions between which it operates. The standards do not mandate which functions should be co-located, as this depends on the scale of the application, and a single device may contain several functions.

Successful execution requires a network architecture that can support development, deployment and delivery over an IP backbone-IMS. IMS carries signaling and bearer traffic over the IP layer, functioning as an intelligent 'routing engine' that matches a user profile with an appropriate call handling server and switches the call control over to the designated handler. IMS includes the capability to add, modify or delete sessions in an existing multimedia call, and extends the IP network all the way to the user equipment, enabling the core network to remain access agnostic. Each end-user can have a personalized experience involving simultaneous voice, data, and multimedia sessions.

The 3GPP architecture is split into three main planes or layers, each of which is described by a number of equivalent names: Service or Application Plane, Control or Signaling Plane, and User or Transport Plane. 


\section{Application plane}

The application plane provides an infrastructure for the provision and management of services, and defines standard interfaces to common functionality including: configuration storage, identity management, user status (such as presence and location), which is held by the Home Subscriber Server (HSS), billing services, provided by a Charging Gateway Function (CGF), control of voice and video calls and messaging, provided by the Control Plane.

\section{Control plane}

The control plane sits between the application and transport planes. It routes the call signaling, tells the transport plane what traffic to allow, and generates billing information for the use of the network.

At the core of this plane is the Call Session Control Function (CSCF), which comprises the following functions.

The Proxy-CSCF (P-CSCF): is the first point of contact for users with the IMS. The P-CSCF is responsible for security of the messages between the network and the user and allocating resources for the media flows.

The Interrogating-CSCF (I-CSCF): is the first point of contact from peered networks. The I$\mathrm{CSCF}$ is responsible for querying the HSS to determine the S-CSCF for a user and may also hide the operator's topology from peer networks (Topology Hiding Inter-network Gateway, or THIG).

The Serving-CSCF (S-CSCF): is the central brain. The S-CSCF is responsible for processing registrations to record the location of each user, user authentication, and call processing (including routing of calls to applications). The operation of the S-CSCF is controlled by policy stored in the HSS.

This distributed architecture provides an extremely flexible and scalable solution. For example, any of the CSCF functions can generate billing information for each operation. The Control Plane also controls User Plane traffic through the Resource and Admission Control Subsystem (RACS). This consists of the Policy Decision Function (PDF), which implements local policy on resource usage, for example to prevent overload of particular access links, and Access-RAC Function (ARACF), which controls QoS within the access network.

\section{User plane}

The User plane provides a core QoS-enabled IPv6 network with access from User Equipment (UE) over mobile, WiFi and broadband networks. This infrastructure is designed to provide a wide range of IP multimedia server-based and P2P services.

Access into the core network is through Border Gateways (GGSN/PDG/BAS). These enforce policy provided by the IMS core, controlling traffic flows between the access and core networks.

Within the User Plane the Interconnect Border Control Function (I-BCF) controls transport level security and tell the RACS what resources are required for a call.

The I-BGF and A-BGF Border Gateway Functions provide media relay for hiding endpoint addresses with managed pinholes to prevent bandwidth theft, and implement NAPT and NAT/Firewall traversal for media flows. 


\section{Applications of IMS}

\section{IPTV}

With the Nortel IPTV solution for example, telephony meets television, which meets mobility providing the very most advanced features of both technologies, synergized to allow for uninterrupted downtime while fully in touch with the outside world. IPTV allows users to relax on the sofa while onscreen before them is access to instant messaging, caller ID and the ability to accept, reject, initiate or even redirect incoming calls to another number or even to voicemail. This solution applies to video for all access technologies, whether mobile television, cable or IP. A recent survey of end users by Nortel indicates that approximately 30 percent have expressed interest in subscribing to interactive services such as these

IPTV benefits:

$>$ Operates with various levels of communications management and types of phones.

$>$ Enhances the end-user experience and helps afford uninterrupted television viewing by enabling the user to check the caller ID without leaving the couch and then presenting them with the option to answer or ignore the call or redirect it to voicemail.

$>$ Links with other IMS capabilities, such as click-to-call, instant messaging, video on demand and buddy lists.

Before IMS we had Proprietary interfaces and Separate subscriber databases, authentications, etc. but now with IMS, proprietary interfaces are replaced with open standards with an integrated single sign-on for telephony, multimedia, TV, video while a single authentication, QoS, DRM, security solution are required for telephony, multimedia and IPTV.

The IPTV solution allows subscribers to stay in touch with friends and family while watching their favorite television shows, to identify who is calling and receive or route calls as they wish. They can go to a voice call with the click-to-call feature or immediately initiate an instant message. Consumers can also receive video photos from a caller on their screens. (Nortel, 2006)

\section{Converged Mobility}

IMS Converged Mobility untethers communications from physical boundaries, freeing users to roam, seamlessly, with unbroken service. By integrating traditional wireless technologies with new wireless technologies, it seamlessly improves and provides coverage in areas where cellular coverage is poor, while maintaining features across cellular and WLAN - for example, voice features, SMS and Instant messaging. Most fundamentally and notably, Nortel Converged Mobility for example allows subscribers to use a single device, a common dial-pad and a common address book in cellular, office WLAN or home WLAN environments.

Converged Mobility benefits:

$>$ Improves in-building coverage and increases reachability.

Before IMS, there was no multimedia integration in mobility; but now with IMS, multimedia is integrated which addresses multiple market segments with a single solution. Converged Mobility allows subscribers to be truly mobile and to communicate freely without having to be concerned with specific access types. Communications conform to customers' lifestyles and follow them intelligently as they move (Nortel, 2006) 


\section{Push to Talk}

IMS Push to Talk (PTT) is a standards-based, end-to-end solution that offers mobile, half-duplex voice communication services that combine 'always-on' capabilities with ease-of-use. PTT is a forerunner to peer-to-peer services over IP, for which IMS provides the capabilities and foundation. PoC is the first commercial application based on IMS (NorthStream, 2004).

Push-to-Talk is all about ease of use and ease of access, allowing customers to use a single button to alert a friend or colleague that they'd like to talk, and to then have a conversation without having to dial their phone. It's a personalized "walkie-talkie" experience - personalized in that the Push-to-Talk solution is integrated with other elements of the IMS infrastructure, such as buddy lists and presence features.

An expansion of the Push-To-Talk service is Push To See and eventually Push To X (anything) involving content-sharing, location-based services and IMS applications across a number of different devices truly enabling enhanced real-time communications.

Push to Talk benefits:

$>$ Increases Average Revenue Per User.

$>$ Enhances end-user convenience with a "walkie-talkie" experience, with the party being dialed just a single button push away (Nortel, 2006)

\section{Web/Audio/Video Conferencing}

IMS enables network operators to combine web, audio, and video in sophisticated conferencing solutions. With Motorola's Web/Audio/Video Conferencing, conference participants can view presentation materials while listening to the audio of a conference, hold simultaneous private text conversations, all while a conference moderator controls addition, deletion and mute status of participants.

\section{Instant Messaging services}

Instant Messaging is a communication service that allows end-users to send and receive messages instantly. Instant Messaging is well known in today's Internet community. IMS will bring the same service experience to the mobile world, including interoperability without the need for legacy infrastructure.

\section{Full Duplex Video Telephony}

With the advent of multimedia sessions running over IP, IMS makes it possible to create true, full duplex video telephony sessions on handsets. For businesses and geographically dispersed families, this means there can finally be a videophone solution that does not depend on proprietary networks and equipment and is mobile. Operators can profit from the ability to offer a costeffective solution for which demand has never been satisfied effectively by past solutions.

The various applications discussed above enable operators/service providers to quickly reap the benefits of IMS deployment. These IMS services have become increasingly popular both with wireline and wireless service providers as they have been designed to increase carrier revenues, create an open standard based network while delivering integrated multimedia services.

\section{Benefits of IMS}

MNOs deploying an open, flexible, and integrated IMS Architecture stand to gain considerable competitive advantages. 


\section{Operator's Benefits}

\section{Time-to-market}

The standardization of both the communication protocol between the different elements and also of the interfaces and functions provided by the common services significantly reduces the Timeto-Market for rolling out new IMS Services and provides MNOs the freedom to experiment with new differentiated services.

\section{Lower costs}

IMS would provide most of the underlying standard interfaces, components and functionality on behalf of the new service (registration, session setup, security, billing, OAM, etc.). This reduces significantly the complexity of the systems decreasing CAPEX and OPEX, especially in a complex service environment where a large number of services have been deployed. Operators need only one infrastructure to support all types of multimedia services.

\section{Best of breed}

IMS open interfaces and standard protocols gives operators the flexibility of selecting the "best of the breed" component from a vendor rather being locked in with one or a limited number of them.

\section{Value chain control}

Using existing technologies, it is hard to open up MNO networks to third party developers while maintaining some degree of control as well as a consistent user experience. Operators will not only control the network access but also the management of multimedia sessions, the application and services provided, the charging and the content, while still being able to selectively delegate the development of new applications to third parties.

\section{Integrated, interoperable services}

Because IMS infrastructure offers open and standard interfaces to $3^{\text {rd }}$ party application developers, services are easily, transparently and inexpensively integrated in the MNO's network by using common enablers such as charging systems, thus significantly reducing integration issues. The fact that IMS interconnects with already existing carrier infrastructure like mobile and fixed CS networks makes the transition path to the IMS solution even more attractive.

\section{End-user Benefits}

End-users will be offered a wider variety of new services with a richer user experience. Some of the benefits of these services will include:

\section{Integrated rich media}

End-users will have the ability to use more than one type of media: e.g. text, audio or video in a single call and simultaneously as well.

\section{Personalized communications}

The end-user will be able to indicate to the network the preferred medium to be reached, according to who is calling, etc. 


\section{Roaming}

The IMS architecture addresses roaming issues, thus enabling end-users to move across MNOs and be able to use all IMS services, as if they were on their home network. Roaming translates into increased revenue for MNOs.

\section{IP services}

End-users will have the opportunity to choose from a plethora of services that they will be able to enjoy in both the wireless and wireline environments. IMS services include non-real time, near real-time and real-time types of services.

\section{Mobile-fixed inter-working}

End-users are able to transparently communicate with other end-users regardless if they are in an IMS or CS telecom network and use traditional telecom CS and IMS services (3G Americas).

\section{Identified Limitations of IMS}

Prediction for the future rests on some sort of evidence or established trend which, with extrapolation, clearly takes us into a new realm. IMS opens up new perspectives for network operators. But several technical and business challenges have to be faced in order to enable the wide adoption of this promising technology. Moreover, IMS has to solve its inherent contradictions: it relies on IP technologies allowing free communication but aims at controlling IP services.

\section{Business issues}

IMS leads networks operators to play a central role in service distribution. This involves that carriers will have to obtain content. The role of the operators in the billing of services provided by third parties has also to be clarified.

With IMS a single customer may subscribe to services from several providers. IMS therefore leads network operators into a competition with players of the Internet world. The decision to deploy IMS is strategic. Network operators may choose an early deployment scheme in order to take advantage of the higher prices charged to early adopters. In this pioneering strategy they would open the way for their competitors and take significant risks. Alternatively, a network operator may wait in order to reduce his investment costs and learn from his competitors failures. But he would face several competitors having better market experience.

As a conclusion, the decision to deploy IMS is more of a strategic decision than a technological decision.

\section{Technical issues}

The end-to-end model adopted in IMS introduces several technical challenges, for example concerning QoS, privacy and billing. The main technological issue is related to interoperability. IMS mixes the points of view of IP, wireline telephony and mobile network operators. Moreover, it introduces new networking paradigms and provides specifications, not implementation-ready solutions. Finally, it uses some recent protocols like Diameter that have not been widely deployed. For all these reasons, interoperability may be difficult to achieve in IMS networks. The TISPAN IMS is designed to be access agnostic. One of the main motivations for IMS is to enable the delivery of real time multimedia services using IP related technologies, but IMS has to manage the different access related constraints imposed by heterogeneous access technologies (e.g. handover in radio access networks). In particular, this makes the establishment of end-to-end QoS guarantees quite difficult. 
Moreover, mobile wireless devices have limited functions, are usually related to a single user and are controlled by the network operator, whereas fixed wired terminals are powerful and controlled by the user. These differences have to be taken into account for authentication (e.g. using SIMbased secure access) and security related operations, for example. IMS aims at providing multimedia services with unified network architecture.

Again, although comprehensive, the 3GPP IMS architecture does not address all of the necessary architecture domains, including those described in this paper. Defining the architecture for these and all other domains is vital if the promises of IMS are to be realized (Weibel et al, 2006).

Additional issues are currently or need to be addressed in the standards, for example: universal service obligation, number planning, lawful intercept, number portability, reliability and voice quality, emergency services, inter-carrier compensation and data protection.

Last but not least, there is a risk that consumers will not readily accept the privacy model related to IMS (Bertran, 2007).

\section{Moving Forward}

The IP Multimedia Subsystem is a key advance towards VoIP SIP-based service, and the next logical step in the evolution of the core network. IMS delivers a new level of capability for operators seeking to meet the demands of their end-users for fresh applications, innovative services, multimedia experiences, enhanced mobility, broad access, and more ubiquitous communication.

Vendors believes IMS not only represents a step forward for evolving networks, but a giant leap toward operator success in an increasingly competitive marketplace. Significant investment has been made in the core network infrastructure business, from handsets, to RANs, to core network components, and finally to service delivery and support structure. Interoperability standards and open architectures has been developed for the industries, all with the aim of addressing the needs of network operator customers, end-to-end, development through delivery. All that experience is brought to fruit in IMS, a solution that delivers the reliability, capacity, and flexibility operators will need to take their networks to the next level.

Meanwhile anticipated factors that will have a significant influence on the direction of IMS are: Government legislation, Consumer pressure and Competitive pressure among operators (Cumming, 2005).

\section{References}

3G Americas. (2004). IP multimedia subsystem (IMS) overview and applications.

Bertrand, G. (2007). The IP multimedia subsystem in next generation networks.

Cumming, J. (2005). Session border control in IMS.

Motorola IP Multimedia Subsystem - White Paper. (2005). Retrieved from www.motorola.com/networkoperators

Mangini, D. (2006). IP multimedia subsystem (IMS): Driving new business models and opportunities.

MetaSwitch Networks. IMS architecture.

Nortel IP Multimedia Subsystem (IMS) Solution. (2006). Deliver converged multimedia services across any access or device.

NorthStream. (2004). Overview and comparison of push-to-talk solutions.

Telecom Report. (2005). IMS Guide:- Light Reading - IP \& Convergence 
Weibel, X., Gourraud, C., Polette, O., \& Longmore, L. (2006). IBM IP Multimedia Subsystem Solutions. Service Architecture for 3GPP IP Multimedia Subsystem - the IBM and Swisscom Proof-of-Concept Experience.

\section{Biographies}
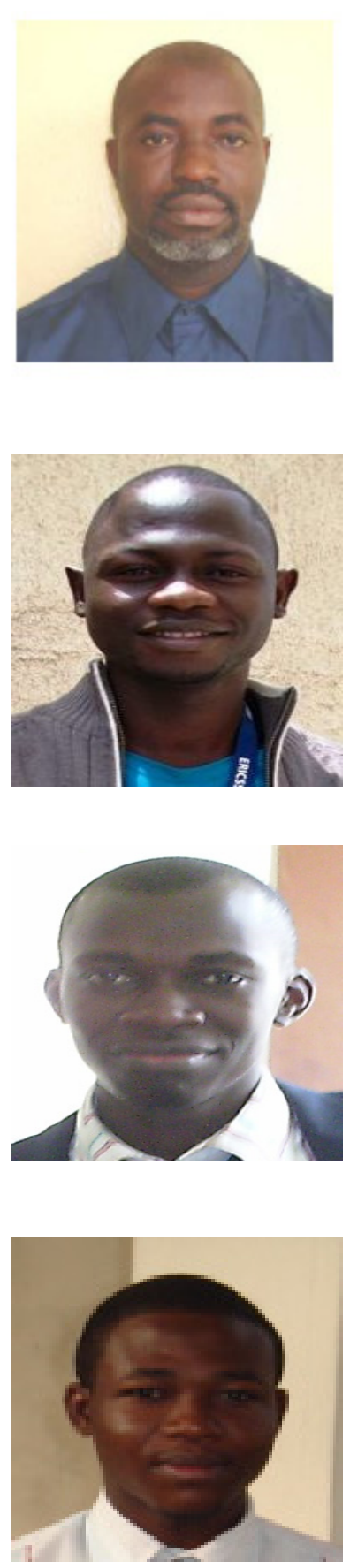

Awodele Oludele ( $\mathrm{PhD})$ is presently the head of Computer Science and Mathematics department, Babcock University. He is a graduate of the University of Ilorin, Nigeria. He has had several years of experience in teaching computer science courses at the university level. He is a member of the Nigeria Computer Society and the Computer Professional Registration Council of Nigeria. His areas of interest are Computer Architecture and Artificial Intelligence. He has published works in several journals of international repute.

Jegede Olawale works with LM Ericsson Nigeria as a Transmission Engineer with bias for IP based solutions. He is a graduate of Babcock University, Nigeria where he obtained a first class degree in Computer Engineering Technology. He is a Cisco Certified Network Professional. His areas of interest include: Telecommunications and Networking, Digital Electronics, Artificial Intelligence and Control. He has published works in journals of international repute.

Onyenweaku Edward works with New Horizons Nigeria Ltd (a Leading World IT firm) as a Consultant/Instructor. He is a graduate of Babcock University, Nigeria where he obtained a first class degree in Computer Engineering Technology. He is a Cisco Certified Internetwork Expert (CCIE-Written) and is presently preparing for his Lab. He is widely traveled and has also been to Cisco Lab in India. His areas of interest include: Inter-Networking, Telecommunications, Information Technology and Digital Electronics.

Shokunbi Opeyemi works as the IT Co-coordinator/Engineer with Nestle Nigeria PLC. He is a graduate of Babcock University, Nigeria where he obtained his first degree in Computer Engineering Technology. He is a Cisco Certified Internetwork Expert (CCIE-Written) and is presently preparing for his Lab. His areas of interest include: Information Technology, Networking and Telecommunications. 